\title{
Battlefield Advanced Trauma Life Support (BATLS)
}

\section{CHAPTER 7 ABDOMINAL INJURIES}

\section{AIM}

0701. On successfully completing this topic, you will be able to:

- Identify casualties who have sustained abdominal injuries.

- Recognise the differences in patterns of abdominal injury based on the history and mechanisms involved.

- Establish management priorities and institute appropriate treatment.

0702. Specifically, you will be able to:

- Describe the anatomical regions of the abdomen.

- Recognise abdominal injury.

- Recognise the difference in injury pattern between blunt and penetrating injury.

- Identify the signs indicative of intraperitoneal, retroperitoneal and pelvic injury.

- List the diagnostic procedures specific to a casualty with abdominal injury.

- Understand the use of diagnostic peritoneal lavage.
- Understand the role of Focused Abdominal Sonography for Trauma (FAST).

\section{INTRODUCTION}

0703. You must correctly identify those casualties who have sustained abdominal injury and require surgery. This requires a high index of suspicion. Unrecognised abdominal injury frequently results in death that could have been prevented, both in peace and war. In young athletic people, such as soldiers, there may be initially no apparent physical signs. As many as $50 \%$ casualties with significant intraperitoneal haemorrhage will have few or no signs when assessed at Role One. These casualties have non-compressible haemorrhage and require urgent surgical intervention.

\section{The abdominal cavity is a silent reservoir for major blood loss.}

0704. Casualties presenting with penetrating abdominal injury will pose little difficulty. Always assume that visceral injury has occurred. In casualties presenting with blunt injury, the history is often the most vital

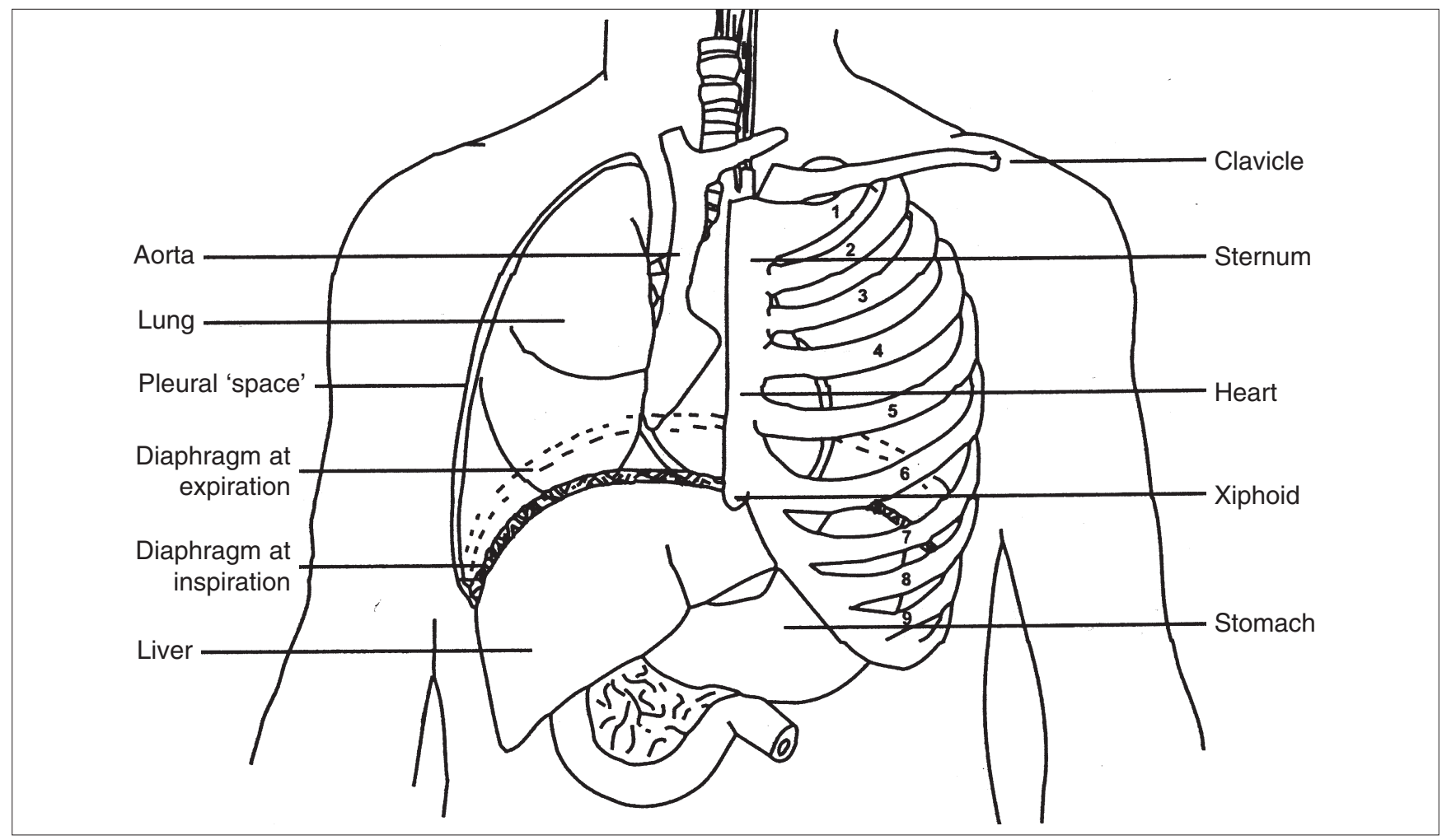


indicator of abdominal trauma. You must assume that the casualties who have sustained significant deceleration have suffered intraabdominal trauma.

\section{Anatomy}

0705. The abdomen has three distinct anatomical compartments - intraperitoneal, retroperitoneal and the pelvis. The intraperitoneal cavity can be further divided into intrathoracic and abdominal components. The intrathoracic abdomen is that portion protected by the lower rib cage and includes the diaphragm, liver, spleen, stomach and transverse colon. The diaphragm can rise to as high as the fourth intercostal space during full expiration (see Fig 7-1), putting these abdominal viscera at risk, particularly from thoracoabdominal penetrating injury. In blunt injuries resulting in lower rib fractures you should be suspicious of damage to the liver or spleen. 0706. The retroperitoneal component contains the aorta, inferior vena cava, kidneys, ureters, the ascending and descending colons, the duodenum and pancreas. Injury to these structures is frequently covert and you must have a high index of suspicion. You should note particularly that diagnostic peritoneal lavage may be misleading: it can fail to detect retroperitoneal haemorrhage.

\section{Blunt versus Penetrating Injury}

0707. The pattern following blunt injury is quite different from that of penetrating injury.

- Blunt Injury, particularly following road traffic accidents, falls, or due to a blast, results from rapid deceleration, deforming or crushing which can cause visceral disruption due to compression and shear forces. The structures most at risk are the liver, spleen, kidneys and retroperitoneal duodenum. Seat belt compression can result in injury to hollow viscera such as the small and large bowel; it can also result in spinal injury.

- Penetrating injury may have direct and indirect effects. Low energy-transfer missiles and stab wounds involve structures directly in their path and cause damage by direct laceration. High energytransfer missiles usually cause considerably more damage, not only to organs in their path but also to adjacent viscera. This is particularly so if solid organs such as the liver, spleen and kidney are involved. Energy-transfer and cavitation is much greater in solid as opposed to hollow viscera (see paragraph 0114). The thoracolumbar spine is also at greater risk of injury from high energy-transfer, as opposed to low energy-transfer, missiles.

\section{IMPORTANCE OF HISTORY}

\section{Blunt Injury}

0708. Blunt injury may be overt or covert. Accurate initial assessment may depend on knowing the circumstances leading to the injury. Of particular importance are the time and mechanism of injury; the nature of impact particularly if a soft-skinned vehicle is involved; whether seat belts were worn; and the condition of other victims. Were any other occupants of the vehicle killed or thrown clear? If possible, get a description of the wrecked vehicle; for example, was the steering wheel buckled? This will point to possible chest or upper abdominal injury. In other words, read the wreckage. All these factors are important in assessing whether or not a high energy impact has occurred. Details of earlier life support measures are also important.

\section{Penetrating Injuries}

0709. While accepting as paramount the maxim, treat the wound, not the weapon, you should get as much valuable historical information as you can. This should include the time of injury; the weapon or other munitions involved; how many shots were fired; the casualty's location at the time of injury, for example, inside an armoured vehicle; and the position of the casualty when hit (crouching, prone and so on - these may give a pointer to the track of the missile). Any earlier life support measures carried out should also be determined. ${ }^{1}$

\section{PRIMARY SURVEY AND RESUSCITATION}

0710. The primary survey and life-saving resuscitation, as with all casualties, take precedence. The secondary survey, the specific in-depth assessment of abdominal injuries, must not take place until completion of the primary survey and resuscitation phases.

\section{Remember the A B C D E routine}

0711. A confident clinical diagnosis of abdominal injury is difficult even under ideal conditions. It is even more difficult under austere field conditions. Further, medical attendants working at Role 1 will be junior and relatively inexperienced. In the primary survey, casualties with abdominal injuries will come to light during assessment of $\mathrm{C}$ in the A B C D E assessment. The problem facing the medical officer or Cbt Med Tech will be "Where is the blood?" In many cases there will be no hard physical signs on abdominal examination that will answer the question. The possibility of an abdominal injury should always be considered. Early evacuation on suspicion alone is totally appropriate. Delay until signs become 
obvious will result in mortality rates over $90 \%$ : this must be avoided. Your task is to answer the question "is the casualty bleeding within the abdomen?". Later, it is the surgeon's task to answer "what is bleeding?".

0712. Additional procedures in the resuscitation phase, depending on the role at which the casualty is treated, are:

-Venous access. Refer to the shock lecture for recommendations on the siting of venous access cannulae. Withdraw blood from one of the venous access cannulae and either send it with the casualty on evacuation or send it to the laboratory. Appropriate immediate tests include full blood count, haematocrit, group and crossmatch and amylase.

- Fluid Resuscitation. Refer to the algorithm on fluid resuscitation in the shock lecture (table 5-2). The overriding principle is to stop the bleeding by early evacuation to a surgical centre. Injudicious use of IV fluids in a casualty with non-compressible haemorrhage may result in exacerbation of existing bleeding or rebleeding. This is particularly true when managing a casualty in the pre-hospital setting.

\section{SECONDARY SURVEY}

0713. You have already made a rapid assessment of the abdomen during the primary survey, identifying and treating all life-threatening conditions. Now the cause of the threat to the casualty may become evident. Do the examination in a systematic fashion:

- Inspection. Whenever feasible, undress the casualty completely. Examine the front of the abdomen, the chest, pelvis and thighs. Turn the casualty fully, log-rolling if necessary, and examine the back. You are looking for open wounds, bruising and obviously swelling. Examine the perineum and do a rectal examination at the same time.

- Palpation. This can yield subjective as well as objective evidence of intra-abdominal injury. Early pain is visceral in origin and poorly localized. Later pain is somatic and leads to involuntary guarding with or without rigidity of the abdominal wall muscles. Ridigity provides unequivocal evidence of peritoneal irritation.

- Percussion. This can yield the earliest sign of peritoneal contamination by blood or faeces, by evincing pain when the abdomen is percussed.

- Auscultation. The presence or absence of bowel sounds may be difficult to determine because of extraneous noise. The presence of a bruit would suggest significant vascular injury, for example arteriovenous (AV) fistula.

- Perineal and rectal examination. This examination may draw attention to:
- Fractured pelvis.

- Ruptured urethra

- High riding prostate.

- Blood at the urinary meatus.

- Scrotal haematoma.

- Vaginal examination. Lacerations from blunt or penetrating wounds or bony spicules indicate serious injury.

- Bladder Decompression. Bladder catheterization, either through the urethra or suprapubic route is both diagnostic and therapeutic. The first aim is to provide a means of monitoring shock therapy by measuring urinary output. The presence of haematuria provides an important indicator of genitourinary injury. It is mandatory that a rectal examination must precede bladder decompression if uretheral injury is a possibility.

- Gastric tube. Passing a gastric tube is both therapeutic and diagnostic. You must decompress the stomach by removing gastric contents, thereby reducing the risk of aspiration, this particularly applies during casualty evacuation when supervision may be less than ideal. The presence of blood in the aspirate suggests upper gastrointestinal injury; this finding may affect priority for evacuation or surgery.

- Screening $X$-rays. Assuming that facilities are available, the only X-rays indicated in the primary survey and resuscitation phases are cross table lateral cervical spine, together with PA of chest and pelvis. Further X-rays should be deferred until the definitive care phase. The taking of $\mathrm{X}$ rays should not delay resuscitation.

Note: Rectal and vaginal examinations and urethral catherisation should only be performed by an appropriately trained medical officer at a static, well equipped medical facility.

\section{A positive abdominal examination points to further action although a negative examination does not preclude significant injury. Repeated assessments are typically required and medical officers in the forward areas may evacuate casualties on the basis of history alone or a high index of suspicion.}

\section{CRITICAL DECISION MAKING}

\section{Blunt Injuries}

0714. In many cases, the decision to evacuate the casualty early will be self-evident, (a penetrating injury in a shocked casualty demands priority 1 evacuation). Other casualties with significant abdominal injury may not be so obvious. A finding of bruising of the abdominal wall, lower thoracic injury, obvious pelvic fracture or other positive physical findings may indicate the need for early evacuation. 
0715. Within field surgical facilities, diagnostic peritoneal lavage (DPL) may yield valuable information particularly if the casualty has suffered severe multi-system injuries or is unconscious following blunt trauma and cannot give a history.

\section{DPL is an operative procedure and should only be performed by the surgeon in charge of the casualty. Placement of a nasogastric tube and bladder catheter are mandatory before DPL.}

0716. Diagnostic peritoneal lavage is contraindicated if there are obvious signs that indicate the need for laparotomy. Relative contraindicators include previous abdominal surgery, morbid obesity and advanced pregnancy. Cirrhosis or established preexisting coagulopathy, possibly present if dealing with civilian casualties, are also relative contraindications.

0717. The indications for diagnostic peritoneal lavage are: history of severe blunt thoracoabdominal injury, altered pain response (that is, reduced level of consciousness), unexplained hypovolaemia, multiple injuries, or if a casualty is not available for reassessment of the abdomen - such as being under anaesthesia during prolonged orthopaedic or neurosurgical procedures.

0718. A positive diagnostic peritoneal lavage result is indicated by bloody aspirate, obvious faeces, bile or small bowel contents appearing in the catheter, or lavage fluid appearing through a chest drain or bladder catheter. A positive result is also indicated by laboratory findings that show greater than $100,000 \mathrm{rbc} / \mathrm{mm}^{3}$, greater than 500 $\mathrm{wbc} / \mathrm{mm}^{3}$, amylase in excess of $175 \mathrm{IU}$ in the aspirate, or the presence of vegetable fibres.

\section{Penetrating Injuries}

0719. Decision making in the forward areas is usually easy. On the battlefield there is no place for conservative management of penetrating wounds - all should be evacuated early with the highest priority.

- Gunshot or fragment wounds. Early laparotomy is mandatory even in an apparently stable casualty.

- Stab wounds. These are relatively uncommon on the battlefield. Whereas a conservative approach may be appropriate in peacetime, it is inappropriate in war. Early evacuation and exploration are mandatory.

- Lower chest wounds. The lower chest lies between the nipple line (fourth/fifth intercostal space) anteriorly, the tips of the scapulae posteriorly (seventh intercostal space), and the costal margin. Penetrating wounds to this region are likely to involve abdominal viscera. The incidence of significant organ injury has been estimated as 15 to $25 \%$ after stab wounds and 45 to $50 \%$ after bullet or fragment wounds. On the battlefield, you should assume abdominal injury in all cases and evacuate to the nearest surgical facility.

- Flank and back wounds. Penetrating wounds in this region may involve the retroperitoneum and can be notoriously silent. A missed retroperitoneal wound to the colon or duodenum can be fatal. The incidence rates for visceral injuries are:

- Back penetration: 5 to $15 \%$

- Flank penetration: 20 to $30 \%$

\section{GENITOURINARY TRACT INJURIES}

0720. You should assume genitourinary injury in all casualties following blunt decelerating injury or penetrating wounds entering the peritoneal or pelvic cavlities. The absence of haematuria does not exclude injury to the genitourinary tract. If the injury is obvious or the index of suspicion indicating genitourinary tract injury is high, early evacuation is necessary.

\section{Blunt Injuries}

0721. Back and loin contusions, haematomas or ecchymosis found during the secondary survey point to possible underlying renal injury; associated fractures of the lower ribs posteriorly increase the probability.

0722. Perineal haematomas and pelvic fractures indicate bladder or urethral injury until proved otherwise. Inability to void urine, or blood at the meatus is absolute evidence of injury. Anterior urethral injury is associated with straddle impact and is usually isolated to that part of the urethra.

\section{Penetrating Injuries}

0723. Penetrating injuries by bullets or fragments involving the back, loin or pelvis indicate a probability of urological injury. Do not rely on finding haematuria.

\section{Aids to Diagnosis}

0724. In the forward areas, a high index of suspicion is essential and casualties should be evacuated early. At role 3 it may be possible to perform a limited range of investigations if time and the casualty's condition permit.

- Intravenous pyelography (IVP). High dose intravenous bolus injection of a suitable contrast medium may give valuable information on renal anatomy and function. For example, unilateral nonfunction of a kidney implies serious disruption of the kidney or its blood supply. Delayed films may give additional information.

- Abdominal ultrasound scanning. This may reveal, for example, disruption of renal 
substance, perinephric haematoma, retroperitoneal haematoma and free intraperitoneal fluid indicating the presence of urine or blood, or both.

- Focused abdominal sonography for trauma (FAST). FAST is an emerging technology which may be performed as an adjunct to the primary survey in contradistinction to normal abdominal ultrasound scanning, which is a secondary survey activity taking place in the radiology department of a field hospital facility. It involves the use of a hand held scanning device and may be utilised at all roles of medical care, provided appropriately trained and skilled personnel are available. In trained hands, FAST may be used to detect blood in the pericardial sac, free blood or fluid in the peritoneal cavity, assess the integrity of solid organs such as liver, spleen or kidney and may also detect the presence of a disrupted bladder.

Note: The gold standard for detecting free blood in the peritoneal cavity remains diagnostic peritoneal lavage (DPL). The utility of FAST as an aid to diagnosis in the field has recently been trialled in Kosovo ${ }^{2}$.

- Urethrography. This can be performed by securing a small (12 French gauge) urethral catheter in the meatal fossa and gently instilling contrast medium. Subsequent Xrays will reveal any urethral tear.

- Computed tomography (CT). This investigation, when available, will play a significant role in planning definitive surgery following complex genito-urinary injury. Mobile CT scanners are planned for Role 3 facilities.

\section{PELVIC INJURIES}

0725. Pelvic fractures in war are commonly associated with high energy blunt trauma causing widespread disruption. Three mechanisms are recognised:

- Anteroposterior compression. This typically results from a pedestrian/vehicle impact, motorcycle accident or crush injury following a fall from a height of greater than $3.5 \mathrm{~m}$. Disruption of the pelvis occurs at multiple sites, causing widening of the pelvic ring and may be associated with catastrophic haemorrhage.

- Lateral compression. This mechanism of injury is associated with motor vehicle side-impact crashes and motor cycle accidents. Injury is usually to one hemipelvis on the impact side, which is disrupted and internally rotated. Major bleeding is uncommon.

- Vertical shear. This mechanism is associated with falls from a height, including parachute accidents and results in shearing of the hemipelvis in the vertical plane. Widespread bony and soft tissue disruption is a feature resulting in major haemorrhage.
0726. Casualties with abdominal injuries associated with pelvic fractures have a high mortality rate and merit special attention. Major haemorrhage is usual in battlefield pelvic injuries. Such haemorrhage is difficult to control and is time consuming and resource intensive to manage. Mixed arterial and venous haemorrhage from large bones and bulky muscle is the underlying problem. Additionally, numerous large, thin-walled veins and major arteries lie within the pelvic cavity and are readily disrupted. Mortality rates associated with open pelvic fractures exceed $50 \%$ in peacetime and are significantly higher on the battlefield.

0727. Major pelvic injury is life-threatening and should be detected in the primary survey; its presence will be confirmed during the secondary survey. Key signs include open wounds in the pelvic region, bruising (particularly in the perineum), and pain or grating on gentle pelvic compression. (Do this only once! Done repeatedly it will increase blood loss). Casualties with a major pelvic fracture are often in excruciating pain. $\mathrm{X}$ ray examination of the pelvis is one of the three radiological investigations allowed in the resuscitation phase of the BATLS programme.

\section{Initial Management}

0728. This to some extent will be dictated by where the casualty is being managed. Adequate volume replacement is essential at all roles of care, bearing in mind haemorrhage is non-compressible or only partially compressible until pelvic fixation is applied. Class IV haemorrhage will be typical and fluid volumes given intravenously must be realistic. Careful monitoring of vital signs and urinary output after each bolus will aid calculation of further requirements. Following immediate resuscitation, a careful and full secondary evaluation must be performed to determine the presence of other injuries and other sources of blood loss. Pelvic binders should be used to support and compress the pelvic ring before the casualty is evacuated early to a surgical centre (see table 7-2)

\section{Management at Role Three}

0729. Early compression of mobile bony elements is the key to haemorrhage control. External fixation is the method of choice and can be applied rapidly, if necessary by nonorthopaedic surgeons. In hospital, immobilization should continue to be used if external fixation is not available. Civilian techniques such as arterial or venous emobilization are unavailable on the battlefield.

0730. Continuing shock, despite vigorous fluid therapy ${ }^{2}$ and external fixation, should suggest an additional source of haemorrhage, usually intraperitoneal and diagnostic 


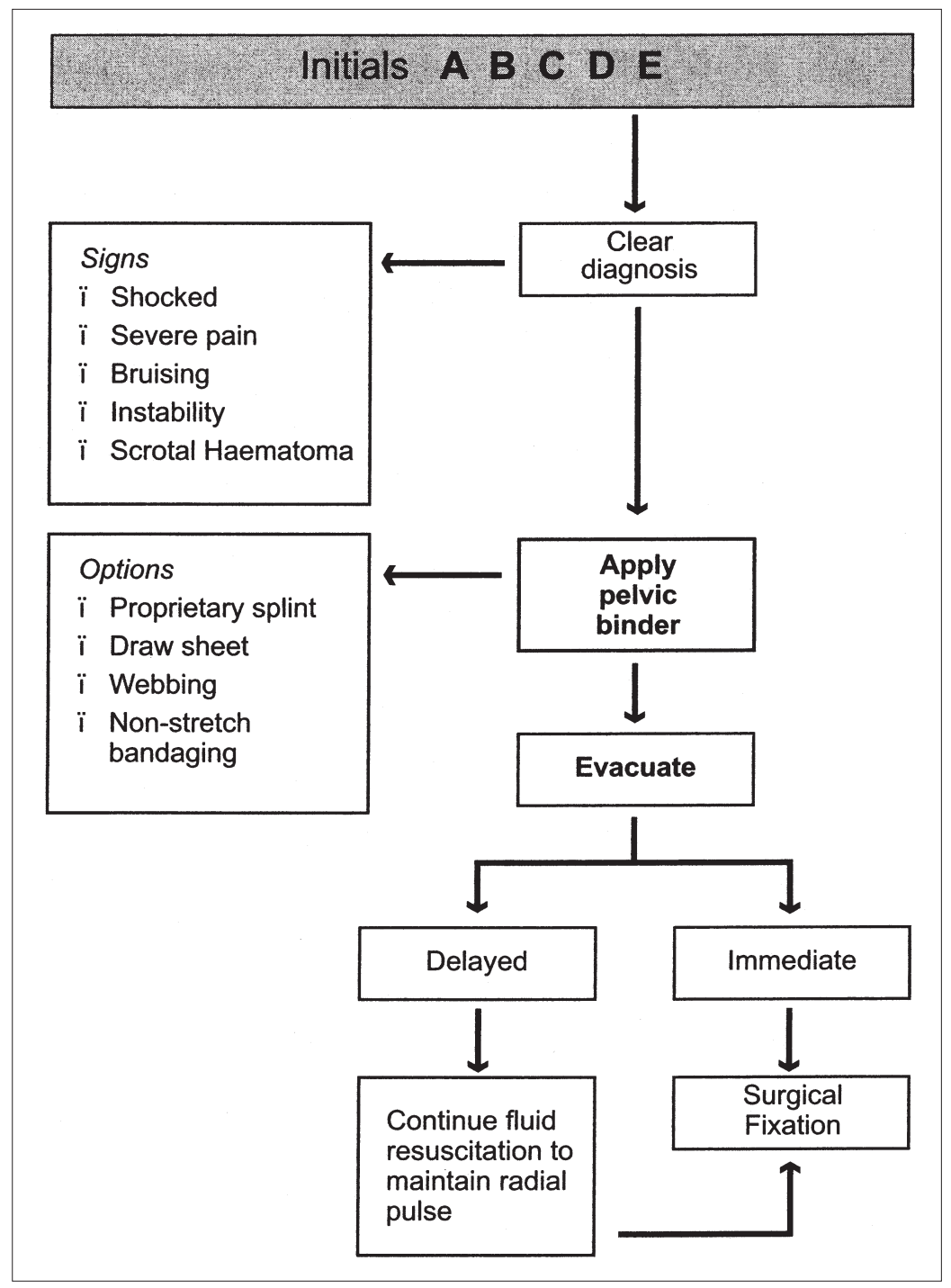

Table 7-2 Management of unstable pelvic fractures in war ${ }^{3}$.

peritoneal lavage by a surgeon should be considered. But there are hazards. Lavage should be performed well above the umbilicus to avoid disruption of a retroperitoneal haematoma. Such disruption may lead to catastrophic and uncontrollable haemorrhage. Whereas negative lavage suggests contained retroperitoneal haemorrhage, a positive lavage needs to be evaluated with caution. In the absence of intraperitoneal haemorrhage, a laparotomy is best avoided as it may lead to uncontrollable haemorrhage by releasing abdominal wall tamponade.

\section{SUMMARY}

- Recognise the possibility of abdominal injury.

- Two major types of abdominal injury may be present in war, blunt and penetrating. In either case, early surgery is essential.

- With blunt injuries, a high index of suspicion is required. Diagnosis in the early stages may be very difficult. Serial examination is required; even then, evacuate early. Aids to diagnosis include: DPL, FAST, CT

- Casualties with penetrating injuries must be given the highest priority for evacuation and surgical evaluation. Penetrating injury to the loin, back, buttocks, lower chest and thighs, should raise suspicion of intraabdominal injury.

- Management of abdominal injury follows normal BATLS protocol and includes:

- Primary survey: Remember the A B C D E routine.

- Resuscitation: This involves:

- Continued oxygenation

- Appropriate therapy for hypovolaemic shock (refer to Chapter 5)

- Continued monitoring of response by assessment of vital signs, aided by additional parameters such as pulse oxymetry and blood gas analysis, when available.

- Placement of nasogastric tube and bladder catheter.

Secondary survey: Following stabilization, but not before, you may proceed to full assessment of the mechanism and location of injury. This demands a full secondary survey and continued monitoring of the casualty. 